\title{
Detection and segmentation of virus plaque using HOG and SVM: Toward automatic plaque assay
}

\author{
Yihao Mao ${ }^{\mathrm{a}}$, Hong Liu ${ }^{\mathrm{a}, \mathrm{b}}$, Rong Ye $\mathrm{e}^{\mathrm{a}, \mathrm{b}}$, Yonghong Shi ${ }^{\mathrm{a}, \mathrm{c}, *}$ and Zhijian Song ${ }^{\mathrm{a}, \mathrm{c}, *}$ \\ ${ }^{a}$ School of Basic Medical Science, Fudan University, Shanghai 200032, China \\ ${ }^{\mathrm{b}}$ Biosafety Level-3 Laboratory, Fudan University, Shanghai 200032, China \\ ${ }^{\mathrm{c}}$ Digital Medical Research Center, Shanghai Key Lab of MICCAI, Shanghai 200032, China
}

\begin{abstract}
Plaque assaying, measurement of the number, diameter, and area of plaques in a Petri dish image, is a standard procedure gauging the concentration of phage in biology. This paper presented a novel and effective method for implementing automatic plaque assaying. The method was mainly comprised of the following steps: In the training stage, after preprocessing the images for noise suppression, an initial training set was readied by sampling positive (with a plaque at the center) and negative (plaque-free) patches from the training images, and extracting the HOG features from each patch. The linear SVM classifier was trained in a self-learnt supervised learning strategy to avoid possible missing detection. Specifically, the training set which contained positive and negative patches sampled manually from training images was used to train the preliminary classifier which exhaustively searched the training images to predict the label for the unlabeled patches. The mislabeled patches were evaluated by experts and relabeled. And all the newly labeled patches and their corresponding HOG features were added to the initial training set to train the final classifier. In the testing stage, a sliding-window technique was first applied to the unseen image for obtaining HOG features, which were inputted into the classifier to predict whether the patch was positive. Second, a locally adaptive Otsu method was performed on the positive patches to segment the plaques. Finally, after removing the outliers, the parameters of the plaques were measured in the segmented plaques. The experimental results demonstrated that the accuracy of the proposed method was similar to the one measured manually by experts, but it took less than 30 seconds.
\end{abstract}

Keywords: Plaque assay, HOG, SVM, local adaptive image segmentation

\section{Introduction}

Viral plaque is a visible structure formed by phage infection and propagation within a cell. Reliable segmentation of plaque from the image of a Petri dish is an essential task in many virological and immunological studies, and it is required for subsequent plaque assay such as detection, separation, purification of phage, and phage counts [1]. Particularly, the parameters such as the number, diameter, and

\footnotetext{
${ }^{*}$ Corresponding author: Yonghong Shi, Digital Medical Research Center, Shanghai Key Lab of MICCAI, Shanghai 200032, China. Tel.: +86 21 54237181; Fax: 86-21-54237797; E-mail: yonghong.shi@fudan.edu.cn.

Zhijian Song, Digital Medical Research Center, Shanghai Key Lab of MICCAI, Shanghai 200032, China. Tel.: +86-2154237054; Fax: 86-21-54237797; E-mail: yonghong.shi@fudan.edu.cn.
}

0959-2989/14/\$27.50 @ 2014 - IOS Press and the authors. 


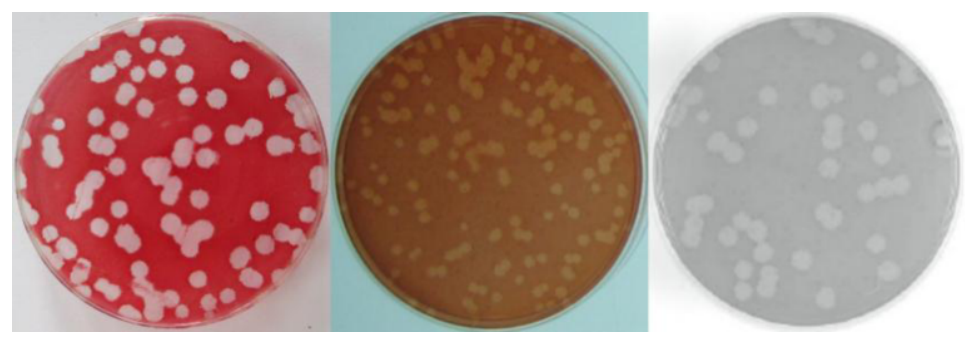

Fig. 1. Illumination of the image of Petri dish for plaque assay.

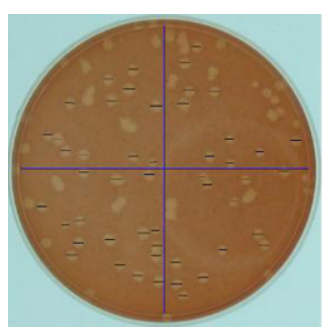

Fig. 2. Illumination of how the diameters of plaques are measured manually.

area of plaques are needed to accurately measure for further comparison or classification of plaques. Figure 1 shows three representative images of Petri dishes for plaque assay, photographed by different virologists $[2,3]$. The typical plaque developed by a phage is the round and relatively bright region in the image of the Petri dish. However, incomplete or clustered plaques make plaque segmentation a difficult task. Complications also arise when plaques are juxtaposed or connected to one another, increasing the rate of over-segmentation or under-segmentation. Furthermore, common parameters like number, diameter, and area of plaques are still measured manually. Figure 2 shows how a virologist measures the diameters of plaques [2,3]. Each short blue line representing the diameter of a virus plaque, which is manually drawn on each plaque, is measured. The long blue line indicating the diameter of the Petri dish is also drawn for estimating the diameter ratio between the plaque and Petri dish. During the stage of phages infecting cells, the virologist faces dozens of images of Petri dishes and measures the plaques one by one. This is inarguably a tedious, error-prone and time-consuming process. Therefore, an automatic plaque detection, segmentation, and measurement system is urgently needed.

Numerous methods are described in literature, among which the grey level threshold is the most commonly used method. For example, Kate, et al. suggested using a flatbed scanner to implement the high throughput virus plaque quantitation, where the identification of plaque depends on a manually set threshold [4]. Moorman et al. proposed a segmentation method by using the Otsu threshold and morphological transformation on the Petri dish for exacting plaques, but it is sensitive to the low contrast and object reflections [5]. Zhang et al. proposed a similar method by applying a modified Otsu for segmentation of plaque after image pre-processing [6]. Clarke et al. presented an automated threshold method for automated counting of bacterial colonies in a low-cost and high-throughput process [7]. Dahle et al. suggested automatically counting mammalian cell colonies by means of a user intervention to set the needed grey level threshold [8]. Some researchers also report using a grey level threshold algorithm to extract the region of interest (ROI) and a watershed algorithm for colony segmentation. Here, a special image acquisition apparatus is required [9-11]. Other methods, including edge detection [12] and Hough transformation [13], are also implemented in cell colony counting and microscopic cell detection. However, with the increasing demand of fine measurements of plaque, a machine-learning-based technique is desirable for accurate detection and segmentation of plaque.

In this paper, an effective and novel method for the detection and segmentation of plaques is presented by means of histogram of oriented gradient (HOG) features $[14,15]$ and support vector machine (SVM) [16]. This method is comprised of the training and the testing stage. In the training stage, the images are pre-processed for noise suppression. Then, the initial training data is set up by sampling the positive training patches (with a plaque at their centers) and negative training patches (plaque-free) from the training images, and extracting the HOG features from each training patch. Third, the linear 


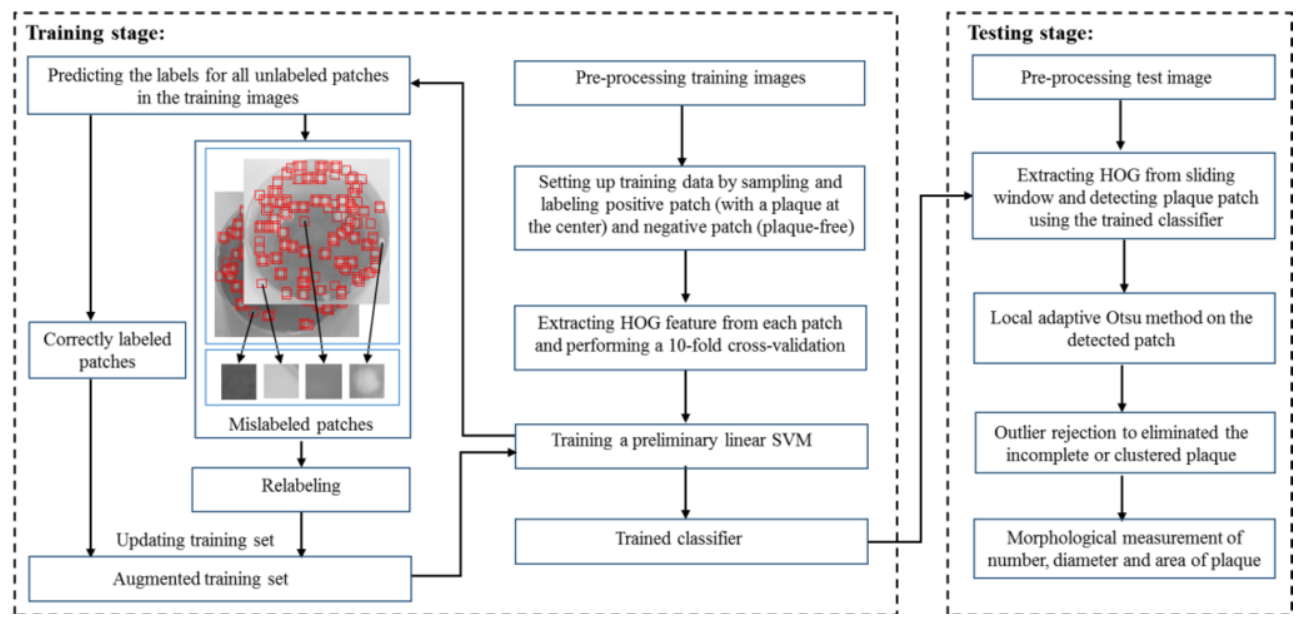

Fig. 3. The pipeline of the proposed automatic plaque assay system.

SVM classifier is trained in a self-learnt supervised learning strategy to avoid possibly missing detection. Specifically, the training set which contains positive and negative patches sampled manually from training images is used to train the preliminary classifier. Then, the preliminary classifier will exhaustively scan the training images by means of sliding window to predict the label for all the unlabeled patches. The mislabeled patches are evaluated by experts and relabeled. Finally, all the newly labeled patches and their corresponding HOG features are further added to the initial training set to train the final classifier. In the testing stage, given an unseen image, a sliding-window technique is utilized to localize the potential patch and calculate the corresponding HOG feature, which is inputted into the trained classifier to predict whether the patch is positive. Then, a local adaptive Otsu method is performed on the positive patches to segment the plaques [17]. Finally, after sifting the segmented plaques (for eliminating outliers) according to the heuristic standards, the parameters, such as number, diameter, and area of the plaque, are automatically measured with precision and speed, compared to those done manually by experts.

This paper is arranged as follows: After presenting technical details in Section 2, and providing extensive evaluation on three data sets in Section 3, the paper is concluded in Section 4.

\section{Methodology}

Figure 3 illustrates the pipeline of the proposed automatic plaque assay system. In the training stage, after pre-processing the training images, the initial training set was readied by sampling the positive patch (with a plaque in the center) and negative patch (plaque-free) from the training images, and extracting the HOG features from each patch. Then the training set was used to train the preliminary classifier which was harnessed to exhaustively search the training images to predict the labels for the unlabeled patches. The mislabeled patches, aka false positive and false negative patches (denoted as 'hard patches'), were diagnosed by experts and relabeled. All the newly labeled patches were further added to the initial training set to obtain an augmented one which, finally, trained the final classifier. In the testing stage, after pre-processing the test image, the HOG features were first obtained in the sliding window, and then inputted into the classifier to predict whether the patch was positive. Once the positive patch was detected, the local adaptive Otsu method was applied to the detected patch for 
segmentation of plaque. Finally, after removing the outliers, such as the incomplete or clustered plaques, the valuable parameters such as number, diameter, and area of the plaques were automatically measured in the remaining segmented plaques.

\subsection{Pre-processing image and extracting HOG feature}

In this study, the stained Petri dish for the plaque assay was captured as a color or grey image. For standardizing the whole procure, the colorful images were converted to the grey image, and all images were scaled to the same size, i.e., $800 \times 800$, for both efficiency and accuracy. Since there existed strong interference and noise, an image preprocessing method, a pixel-wise adaptive Wiener filter with neighborhoods of 6-by-6, was applied to filter the image. Figures 4(c) and 4(d) show a more smooth image after applying the Wiener filtering on the original image, as seen in Figures 4(a) and 4(b). Then, based on the enhanced image, better HOG features were obtained to distinguish plaque from culture medium.

The HOG, a popular feature descriptor, was first used to calculate the occurrence of gradient direction in the local patch of an image. It captured edge and gradient structures, which are characteristic of local shapes, while it performed photometric and geometric transformations [14]. In this study, the image was denoted as $I(x, y)$ with an image size of $\mathrm{M} \times \mathrm{N}$, where $\mathrm{x}$ and $\mathrm{y}$ were the pixel position. The HOG features were extracted in the following steps [15]:

Step 1: After calculating the gradient $f_{x}(x, y)$ and $f_{y}(x, y)$ along $x$-or $y$ - direction of the image, $I(x, y)$, the orientation $\theta(x, y)$ and the magnitude $m(x, y)$ of each pixel in the image were computed.

$$
\begin{aligned}
& f_{x}(x, y)=I(x+1, y)-I(x-1, y) \\
& f_{y}(x, y)=I(x, y+1)-I(x, y-1) \\
& m(x, y)=\sqrt{f_{x}(x, y)^{2}+f_{y}(x, y)^{2}} \\
& \theta(x, y)=\tan ^{-1} f_{x}(x, y) / f_{y}(x, y) .
\end{aligned}
$$

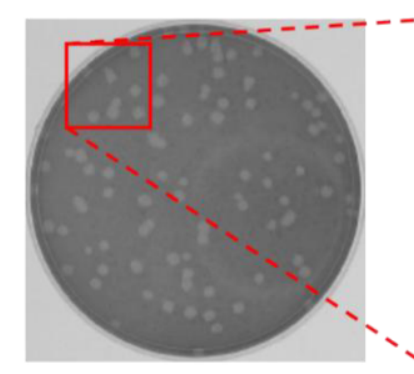

(a)

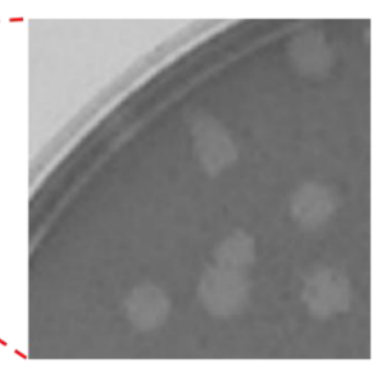

(b)

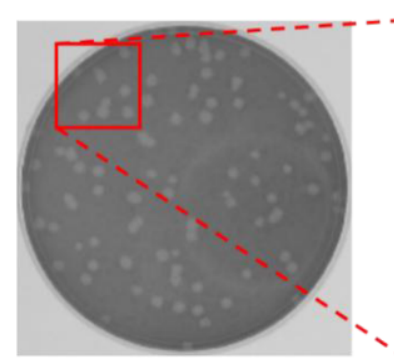

(c)

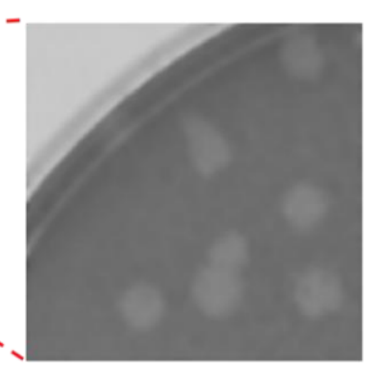

(d)

Fig. 4. Illustration of the effects of the adaptive Wiener filter. (a), (b) Results after converting to greyscale image. (c), (d) Results after applying Wiener filter. 

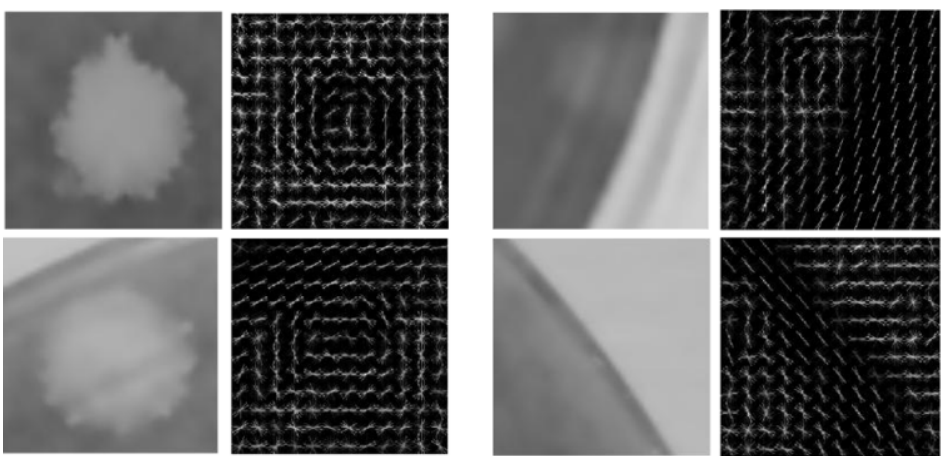

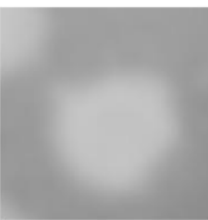

(a)

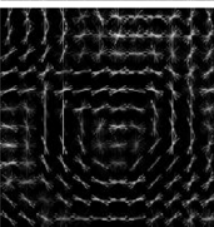

(b)

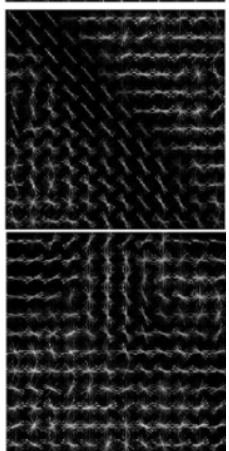

(d)

Fig. 5. Three positive training patches and their HOG features are shown in columns (a) and (b), respectively. Also, three negative training patches and their HOG features are presented in columns (c) and (d), respectively.

Step 2: The image was divided into small connected regions, called cells, and the histograms of gradient directions or edge orientations in each cell were calculated. Each pixel contributed a weighted vote for orientation. In the implementation, each cell size was $4 \times 4$ pixels, and the orientation $(0-180$ degree) was separated into 9 histogram bins equally.

Step 3: The $2 \times 2$ cells were combined into a block whose size was $8 \times 8$ pixels and histogram normalization was performed on the block. Each patch was composed of $6 \times 6$ blocks and the size of patch was $48 \times 48$. The descriptor was the vector of all components of the normalized cell responses from all of the blocks in the patch. Finally, a 4464-dimentional vector, denoted as $u_{k}$, described the feature of the $k$-th patch.

In this way, the HOG features were extracted from both the training and testing patches. Typical examples are shown in Figure 5 to illustrate the pattern of the positive and negative patches.

\subsection{Training linear SVM classifier}

Support vector machine, SVM, is a widely used supervised learning model for data analysis and pattern recognition. SVM finds a hyperplane or a set of hyperplanes to separate data points and maximize the distance to the nearest data point of any class in a high-dimensional space [16]. Here, a linear SVM was adopted to train the data since linear SVM works efficiently with high dimensional data and has relatively less computational complexity compared to other known SVM models [18]. The maximum margin hyperplane, $\Phi\left(u_{k}\right)=u_{k} \cdot w+b$, is found according to the quadratic optimization problem:

$$
\min _{w, b} \frac{1}{2}\|w\|^{2}+C \sum_{k=1}^{K} \xi_{k}, \quad \text { subject to } \Phi\left(u_{k}\right)\left(u_{k} \cdot w+b\right)-1+\xi_{k} \geq 0
$$




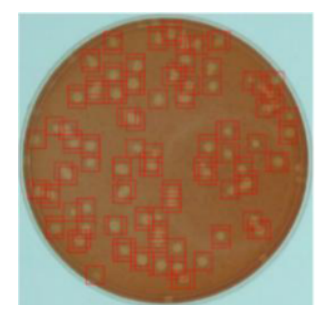

(a)

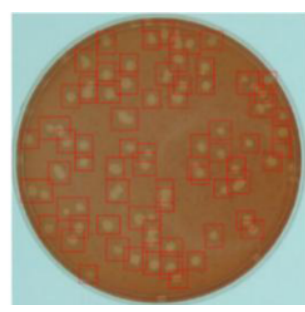

(b)

Fig. 6. Illustration of the detection by the learned classifier. (a) Result using the learnt classifier. (b) Result after performing the window fusion.

where $w$ and $b$ define the hyperplane, $\xi_{k}=\max \left(0,1-\Phi\left(u_{k}\right)\left(u_{k} \cdot w+b\right)\right), C$ is the tradeoff constant, and $K$ is the number of training patches. Then, the classifier is trained as follows:

We also implement SVMs with other kernel functions, such as RBF and polynomial, and compare them with the linear kernel [19]. The RBF kernel is defined as

$$
K\left(u_{k}, u_{k}^{\prime}\right)=\exp \left(\gamma\left\|u_{k}-u_{k}^{\prime}\right\|_{2}^{2}\right)
$$

where $\gamma\left\|u_{k}-u_{k}{ }^{\prime}\right\|_{2}^{2}$ is recognized as the squared Euclidean distance between the two feature vectors while $\gamma$ is a free parameter. For the degree-d polynomials, the polynomial kernel is defined as

$$
K\left(u_{k}, u_{k}^{\prime}\right)=\left(u_{k} \cdot u_{k}^{\prime}+c\right)^{d},
$$

where $c$ is a free constant.

We implement a self-learnt supervised learning strategy to train our classifier. Concretely, the classifier is trained as following. First, patches with plaque at their center were sampled and labeled from each training image as the positive training samples by experts while plaque-free patches were similarly sampled and labeled from each training image as the negative training samples. And all these patches formed the training set. Second, The HOG feature of each patch was extracted as a descripting vector and a 10-fold cross-validation was performed to assess the accuracies (precisions, recalls and AUC). And the preliminary classifier was trained. Third, the preliminary classifier was used to predict the labels for all unlabeled patches sampled by means of sliding window on the training images. The mislabeled patches, aka false positive and false negative patches (denoted as 'hard patches'), were diagnosed by experts and relabeled. Fourth, all the patches were added to the initial training set to construct an augmented one and another round of 10 -fold cross-validation was performed to assess the improvement by adding unlabeled patches. Finally, the final classifier was trained using the augmented training set. Specifically, based on the LIBSVM [18], an easily applied SVM library, a soft linear SVM classifier was trained in the augmented training set. Figure 6(a) shows the detected patches surrounded by red rectangular using the trained classifier.

\subsection{Detecting plaques}

The sliding-window technique was utilized to search exhaustively for positive patches. A fixed-size rectangular window $(48 \times 48)$ was used to scan the image with the stride of 12 rows. The HOG features 
were extracted from the window and inputted into the learnt classifier, and then a prediction whether the patch was positive was returned.

Usually, multiple detections of the same plaque were simultaneously presented. Accordingly, a window fusion method which combined overlapping detections into a single detection was needed. Similar to the method described in [20], the set of overlapping detections were partitioned into disjoint subsets. Then, two detections were classified into the same subset if their overlapping area was larger than $60 \%$ of the area of either detection according to Eq. (6). Finally, each subset yielded a single final detection. Figure 6(b) shows the patches after performing the window fusion.

$$
\frac{\operatorname{Area}(\operatorname{detection}(i)) \cap \operatorname{Area}(\operatorname{detection}(j))}{\operatorname{Area}(\operatorname{detection}(i))}>0.6, \text { or, } \frac{\operatorname{Area}(\operatorname{detection}(i)) \cap \operatorname{Area}(\operatorname{detection}(j))}{\operatorname{Area}(\operatorname{detection}(j))}>0.6
$$

\subsection{Segmenting plaques by locally adaptive Otsu method}

The Otsu method is an automatic image threshold method which computes the optimum threshold separating a grey scale image into two classes so that their combined spread (intra-class variance) is minimal and converts the image into a binary image [16]. Here, a local adaptive Otsu method was performed on each patch, which was predicted as being positive. Because of the relatively small photometric transformation in these local regions, plaques can be well segmented without implementing an illumination correction beforehand. Figure 7 shows the comparison between applying the adaptive Otsu method in each sliding window (Figures 7(a1) and 7(a2)) and applying it in each positive patch predicted by the classifier (Figures 7(b1) and 7(b2)). It was obvious that this method had better performance in segmentation of plaque because the learnt classifier eliminated most of the false positive patches.

\subsection{Rejecting outlier}

Since there were incomplete or clustered objects in the segmented image, heuristic outlier rejection rules were adopted to sift plaques. First, a flood-fill algorithm was applied in the segmented binary image to calculate the size, centroid, major axis length, and minor axis length of each segmented plaque. Second, the noising plaques were rejected, such as the objects which were out of the Petri dish because their centers were farther away from the image center than the radius of Petri dish. Third, the detected plaques whose major axis divided by minor axis was greater than 2 were rejected since they were either incomplete or combined with multiple plaques. Fourth, the combined multiple plaques whose area was 2 times more or 0.5 times less than the mean area calculated were removed from the remaining plaques. Fifth, the detected results which meet the standard described in Eq. (7) were removed. This meant that the detected result could be either deformed plaques or false positive results because the shape of the detected result deviated from a circular shape.

$$
\frac{4 \pi \times \operatorname{Area}(\text { plaque in detection }(i))}{\text { Perimeter }(\text { plaque in detection }(i))}<0.7
$$



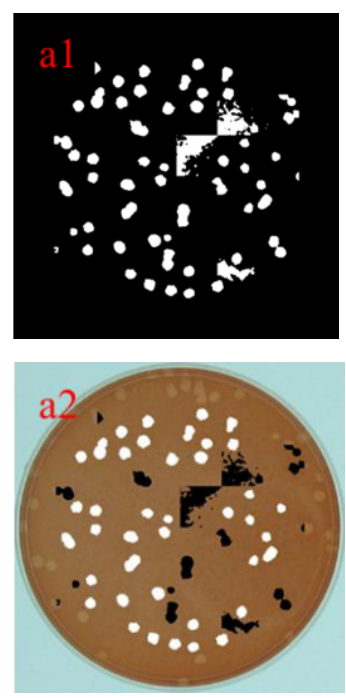

Fig. 7. The segmented results applying the adaptive Otsu method in each sliding window (a1) and the one performing locally adaptive Otsu method on each detected patch by the learnt classifier (b1). (a2) and (b2) are the ones overlaying the segmented results on the original images.
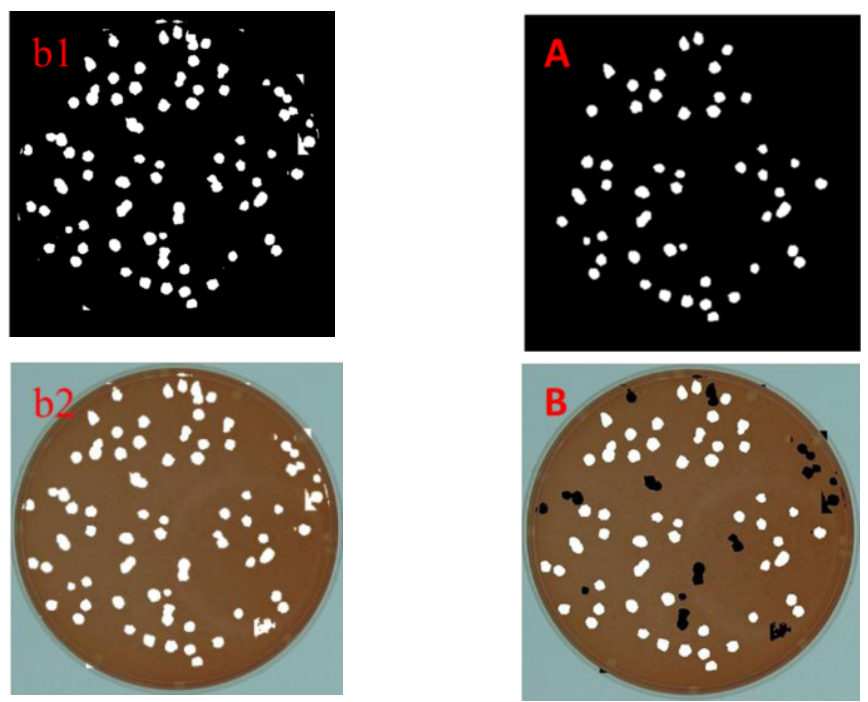

Fig. 8. Illustration of the results after applying outlier rejection in Fig. 7(b1). The segmented result is in (A) which is overlaid in original image (B).

The remaining plaques (shown in Figures 8(A) and 8(B)) were enough to easily and accurately estimate the statistic parameters of virus plaques, such as the number, diameter, and area for further study by virologists and immunologists.

\section{Experimental results}

The proposed method was evaluated by a database which was composed of various images with different image sizes and colors photographed by different virologists. Concretely, three virologists provided their data, which are described as following: The data set 1 contained 8 colorful images with sizes of $787 \times 787$, and the diameters of the plaques of each image were measured by the virologist [2]. Data set 2 included 5 colorful images with sizes of $1803 \times 1803$, and the diameters of the plaques of each image were also measured by a specialist. Data set 3 was a pack of 3 grey images with sizes of $709 \times 709$ and the diameters of the plaques were presented as a relative value to a control image [3]. The manual measurements by the experts can be used as ground truth to evaluate the performance of this method. Here, the results by adaptive Otsu method are provided, based on a sliding window since it is extensively applied in the segmentation of plaque or cells. During the implementation of adaptive Otsu method, the image preprocessing, size of the sliding window, and outlier rejection are kept as the same as outlier method.

With 16 images in the database, they were randomly separated it into two subsets, the training set and the testing set. Each set contained 8 images. This division ensured that both sets contained an equal amount of cases from the above three data sets. Images in one set were detected and segmented with the images in the other set as training set, and vice versa. Then 400 positive patches and 400 negative patches were sampled by experts from 8 training images totally and the corresponding HOG feature for each patch were used to train the classifier according to the method in Section 2. The perfor 

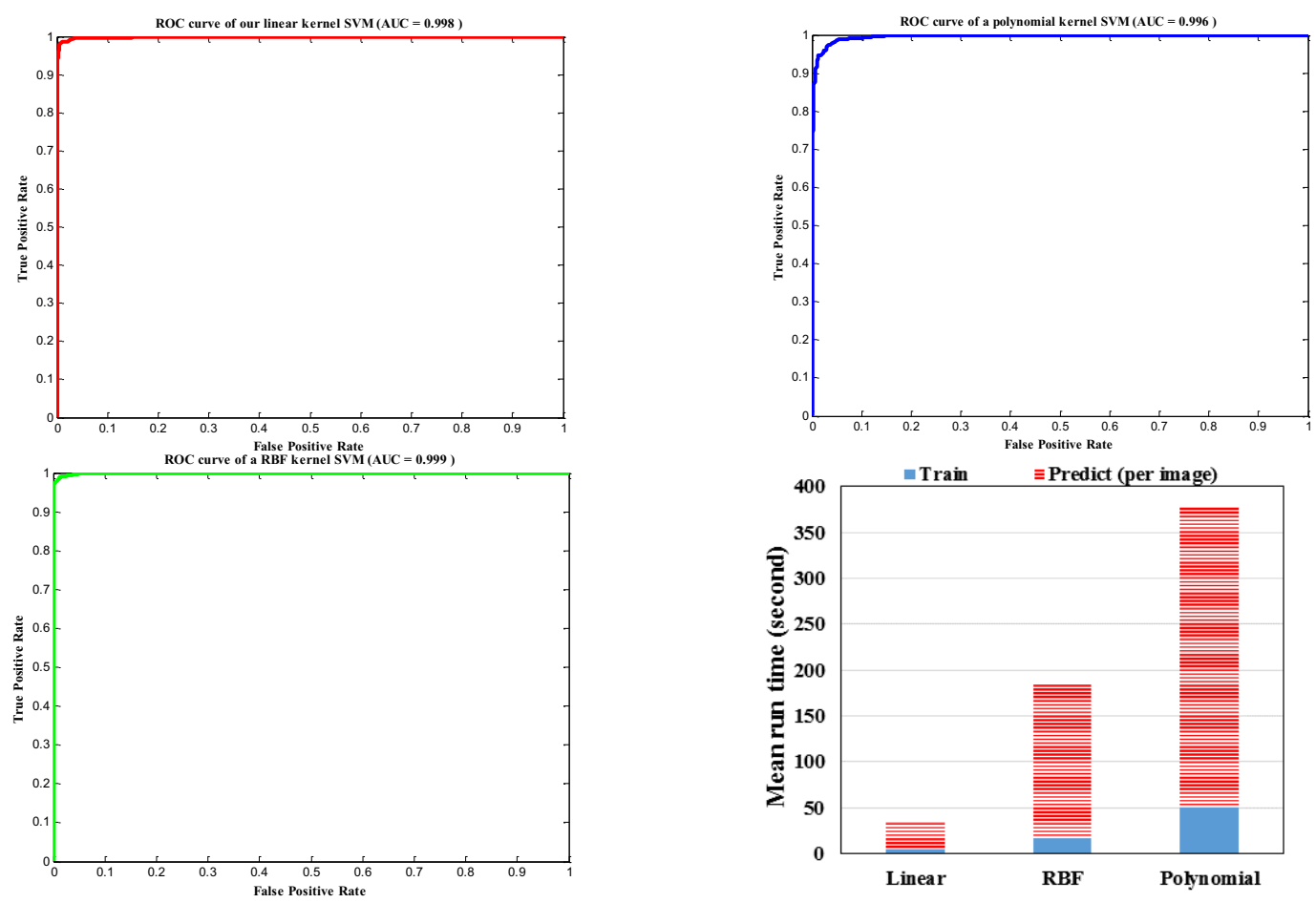

Fig. 9. Illustration of the performance of the learnt SVM classifier with three different kernel functions, such as linear, $\mathrm{RBF}$ and polynomial kernel.

mance of detecting plaque by the classifier and segmenting plaque by the locally adaptive Otsu method was evaluated in the testing set.

The parameters of precision and recall were used to evaluate the method quantitatively and defined as follows:

$$
\text { Precision }=\frac{T P}{T P+F P}, \quad \text { Recall }=\frac{T P}{T P+F N} \text {. }
$$

Here, TP, FP, and FN denote true positive, false positive and false negative, respectively. Also, for showing the reasonability of the method, mean diameter and number of plaque for each image from three data sets were calculated to quantitatively compare with the one manually measured by experts.

The SVMs with different kernel functions, such as linear, RBF and polynomial kernel, were implemented and compared. The parameters of these kernel functions were all of the default values of LIBSVM, where $C=1$ in Eq. (5), $\gamma=1$ in Eq. (6), $\mathrm{c}=0$ and $\mathrm{d}=3$ in Eq. (7) [18].

All the experiments were implemented on an Intel Core i7 2.7-GHZ CPU laptop with 6GB RAM by MATLAB 2013b. Given a trained classifier, the running time from scanning the testing image using sliding-window to outputting quantitative and qualitative results was less than 30 seconds.

Figure 9 shows the ROC curves and the comparison of the running time of the SVMs with three different kernels such as linear, RBF and polynomial kernel, respectively. For example, the AUC values of the classifier with linear, RBF and polynomial kernel were $0.998,0.999$ and 0.996 , respectively while the corresponding time taken for training classifier and detecting the plaque patches in a test im 


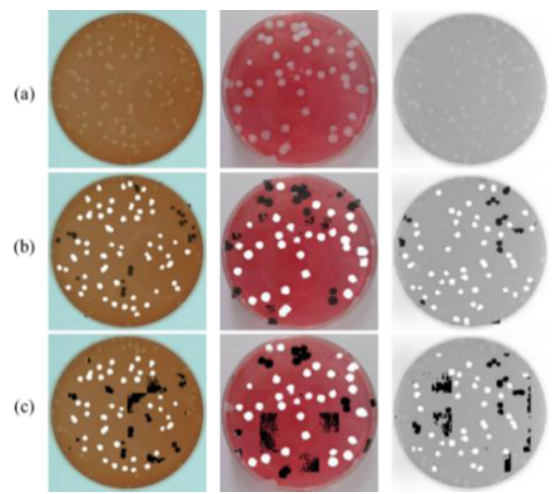

Fig. 10. Segmentation results by this method and a locally adaptive Otsu method, where Row (a) represents three original images; Row (b) is the corresponding segmented results by this method; and Row (c) is the corresponding segmented results by the adaptive Otsu method, where validated plaque is white.

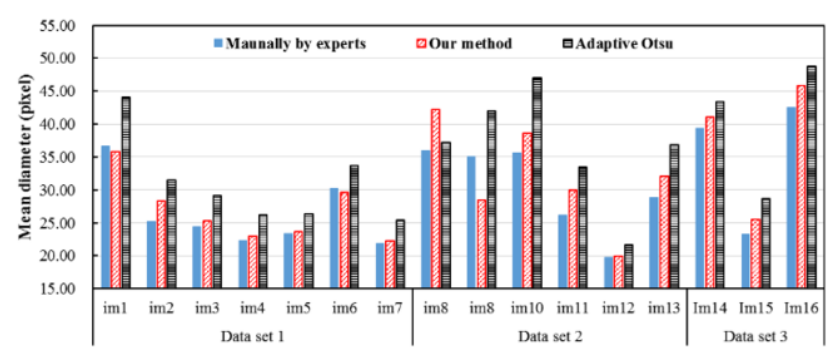

Fig. 11. Comparison of the mean diameter of the plaque for each image from the three data sets by expert manually, this method, and the adaptive Otsu method.

age were $34.86 \mathrm{sec}, 185.00 \mathrm{sec}$ and $377.07 \mathrm{sec}$, respectively. The linear kernel which well balanced the accuracy and efficiency was the optimal choice among three kernels.

Based on the detected patches, Figure 10 visually illustrates the segmentation results by this method. The isolated plaques were accurately recognized in white, and the rejected regions were labeled as black. Here, the original images captured by three virologists, the segmented results by this method, and the locally adaptive Otsu method are shown in the row of Figures 10(a), 10(b) and 10(c), respectively. It is obvious that this method can provide more reliable results because the segmentation was only applied in the positive patches detected by learnt classifier, which removed most of false positive patches. Figure 11 shows the mean diameter for each image from the three data sets by experts manually, this method, and the adaptive Otsu method, respectively. Table 1 shows the mean diameter and standard deviation for each data set by expert manually, this method, and the adaptive Otsu method, respectively. For each image, it can be seen that mean diameter by this method was basically consistent with the one measured by an expert, while the one by the adaptive Otsu method was very different from the one measured by expert. For all images, the mean diameter by our method is 32.07 pixels, which was very similar to the one measured by the expert, i.e., 30.53 pixels, where the mean diameter using the adaptive Otsu method was 35.86 pixels. Regarding the results measured by experts manually as ground truth, Table 2 compares the precision and recall value for the three data sets between this method and the adaptive Otsu method. For total images, the precision and recall values were $96.12 \%$ and $97.28 \%$ for this method, while the corresponding ones by the adaptive Otsu method were $97.42 \%$ and $78.27 \%$, respectively. Table 3 shows the number of plaque in the data set 1 detected by this method and the adaptive Otsu method since only this data set provided the number of plaque of each image. The mean numbers of plaque in data set 1 were counted as 47,44 , and 39 by the expert, this method, and the adaptive Otsu method, respectively. It indicated that this method can find similar numbers of plaque by comparison with the one by the expert. Here, the number of plaque in the first image (im1 in Table 3) was 41 according to the expert, which was very different from the 29 counted by this method. The reason was that this method removed the juxtaposed plaques, which are counted by the expert. The results showed that: (1) The performance of this method was very similar to the one 
Table 1

Comparison of the mean diameter and standard deviation of the plaque for each data set by expert manually, this method, and the adaptive Otsu method (Data layout: mean \pm std. (Unit: pixel))

\begin{tabular}{lllll}
\hline & Data set 1 [2] & Data set 2 & Data set 3 [3] & Total data \\
\hline Manual & $26.32 \pm 5.37$ & $30.24 \pm 6.53$ & $35.03 \pm 10.35$ & $30.53 \pm 7.15$ \\
\hline Our method & $26.86 \pm 4.82$ & $31.88 \pm 8.20$ & $37.47 \pm 10.63$ & $32.07 \pm 7.95$ \\
\hline Adaptive Otsu & $30.91 \pm 6.09$ & $36.36 \pm 7.87$ & $40.30 \pm 8.47$ & $35.86 \pm 8.13$ \\
\hline \hline
\end{tabular}

Table 2

Comparison of the precision and recall value for all of the images in each data set by this method and the adaptive Otsu method when regarding the results measured by experts manually as ground truth (Data layout: mean \pm std. Unit: \%)

\begin{tabular}{l|l|llll}
\hline \multirow{2}{*}{ Precision } & Our method & $98.26 \% \pm 0.83$ & $92.45 \% \pm 3.67$ & $98.57 \% \pm 1.93$ & Total data \\
\cline { 2 - 6 } & Adaptive Otsu & $97.07 \% \pm 2.05$ & $97.56 \% \pm 3.09$ & $98.48 \% \pm 1.31$ & $97.42 \% \pm 2.53$ \\
\hline \multirow{2}{*}{ Recall } & Our method & $96.72 \% \pm 1.24$ & $98.22 \% \pm 1.55$ & $96.91 \% \pm 0.94$ & $97.28 \% \pm 1.52$ \\
\cline { 2 - 6 } & Adaptive Otsu & $81.97 \% \pm 11.08$ & $72.73 \% \pm 15.77$ & $79.27 \% \pm 4.31$ & $78.27 \% \pm 13.56$ \\
\hline \hline
\end{tabular}

Table 3

Number of plaque for each image from data set 1 [2] detected by expert manually, this method, and the adaptive Otsu method.

\begin{tabular}{lllllllll}
\hline Data set 1 [2] & $\operatorname{Im} 1$ & $\operatorname{Im} 2$ & $\operatorname{Im} 3$ & $\operatorname{Im} 4$ & $\operatorname{Im} 5$ & $\operatorname{Im} 6$ & $\operatorname{Im} 7$ & Total image \\
\hline Manual & 41 & 44 & 44 & 50 & 50 & 50 & 50 & 47 \\
\hline Our method & 29 & 48 & 57 & 48 & 50 & 41 & 40 & 44 \\
\hline Adaptive Otsu & 27 & 40 & 38 & 42 & 41 & 46 & 39 & 39 \\
\hline \hline
\end{tabular}

by the expert, and far better than the adaptive Otsu method; (2)This method was enough to accomplish the accurate plaque assay.

\section{Conclusion}

This paper presented a virus plaque detection and segmentation method based on HOG and SVM. After preprocessing the original image by the Wiener filter, the training patches were randomly selected from the images, and the HOG features were extracted from the corresponding training patches. The set of training patches and their corresponding HOG features were used for training the initial linear SVM classifier, which was harnessed to scan the training images exhaustively to predict the labels for the unlabeled patches. The mislabeled patches were diagnosed by experts and relabeled. Then, all the newly labeled patches were added into the initial training set to obtain an augmented one which trained the final classifier to detect the plaque patch in the testing image. Once the plaque patches were detected, a locally adaptive Otsu method was used to segment the plaque from the patch. Extensive experimentation showed that the method was feasible in detecting and segmenting plaques and the performance was satisfied by comparing it to the ones measured by experts manually. In conclusion, this method is adequate for plaque assay analysis and meets the research demands of virologists. In the future, there are plans to enhance the performance of this method by resolving the problems of oversegmentation or under-segmentation. 


\section{Acknowledgement}

This research was supported by the grants from National Natural Science Foundation of Chine grants 60972102,81271670 and 31170786 . This research was also supported by the grants from National Science Talents Bases (J1210041).

\section{References}

[1] H. Lodish, A. Berk, C.A. Kaiser and M. Krieger, Viruses: parasites of the cellular genetic system, in: Molecular Cell Biology, 5th edi., W.H. Freeman, New York, 2000, pp. 137-146.

[2] J. Yang, J. Lv, Y. Wang, S. Gao, Q. Yao, D. Qu and R. Ye, Replication of murine coronavirus requires multiple cysteines in the Endodomain of Spike Protein, Virology 427 (2012), 98-106.

[3] K.R. Hurst, R. Ye, S.J. Goebel, P. Jayaraman and P.S. Masters, An interaction between the nucleocapsid protein and a component of the replicase-transcriptase complex is crucial for the infectivity of coronavirus genomic RNA, Journal of Virology 84 (2010), 10276-10288.

[4] K. Sullivan, J. Kloess, C. Qian, D. Bell, A. Hay, Y.P. Lin and Y. Gu, High throughput virus plaque quantitation using a flatbed scanner, Journal of Virological Methods 179 (2012), 81-89.

[5] M. Moorman and A. Dong, Automated viral plaque counting using image segmentation and morphological analysis, Proceedings of 2012 IEEE International Symposium on Multimedia (ISM'12), 2012, 157-160.

[6] C.C. Zhang, W.B. Chen, W.L. Liu and C.B. Chen, An automated bacterial colony counting system, Proceeding of 2008 IEEE International Conference on Sensor Networks, Ubiquitous and Trustworthy Computing (SUTC'08), 2008, 233240 .

[7] M.L. Clarke, R.L. Burton, A.N. Hill, M. Litorja, M.H. Nahm and J. Hwang, Low-cost, high-throughput, automated counting of bacterial colonies, Cytometry A 77 (2010), 790-797.

[8] J. Dahle, M. Kakar, H.B. Steen and O. Kaalhus, Automated counting of mammalian cell colonies by means of a flat bed scanner and image processing, Cytometry A 60 (2004), 182-188.

[9] K. Claytor, Development and implementation of an efficient automated cell colony and plaque counter, University of Illinois Internal Physics Publication, 2008.

[10] S.D. Brugger, C. Baumberger, M. Jost, W. Jenni, U. Brugger and K. Mühlemann, Automated counting of bacterial colony forming units on agar plates, PloS One 7 (2012), 1-6.

[11] H. Ates, and O.N. Gerek, An image-processing based automated bacteria colony counter, 24th International Symposium on Computer and Information Sciences (ISCIS 2009), 2009, 18-23.

[12] E.D. Cheng, S. Challa and R. Chakravorty, Microscopic cell detection based on multiple cell image segmentations and fusion algorithms, 2nd International Conference on Biomedical Engineering and Informatics (BMEI'09), 2009, 1-7.

[13] J.M. Bewes, N. Suchowerska and D.R. McKenzie, Automated cell colony counting and analysis using the circular hough image transform algorithm (CHiTA), Physics in Medicine and Biology 53 (2008), 5991-6008.

[14] N. Dalal and B. Triggs, Histograms of oriented gradients for human detection, Proceeding of 2005 IEEE Computer Society Conference on Computer Vision and Pattern Recognition (CVPR'05) 1 (2005), 886-893.

[15] P.F. Feizenszwalb, R.B. Girshick, D. McAllester and D. Ramanan, Object detection with discriminatively trained partbased models, IEEE Transaction on Pattern Analysis and Machine Intelligence 32 (2010), 1627-1645.

[16] C.J. Burges, A tutorial on support vector machines for pattern recognition, Data Mining and Knowledge Discovery 2 (1998), 121-167.

[17] N. Otsu, A threshold selection method from gray-level histograms, IEEE Transactions on Systems, Man, and Cybernetics SMC-9 (1979), 62-66.

[18] C.C. Chang and C.J. Lin, LIBSVM: a library for support vector machines, ACM Transactions on Intelligent Systems and Technology (TIST) 2 (2011), 1-27.

[19] M. Mohri, A. Rostamizadeh and A. Talwalker, Kernel method, in: Foundations of Machine Learning, The MIT Press, Massachusetts, 2012, pp. 89-120.

[20] P. Viola and M. Jones, Rapid object detection using a boosted cascade of simple features, Proceeding of the 2001 IEEE Computer Society Conference on Computer Vision and Pattern Recognition (CVPR'01) 1 (2001), 1-511. 